\title{
Ranking of Fuzzy Numbers using Radius of Gyration of Centroids
}

\author{
A. Hari Ganesh ${ }^{1}$, S. Jayakumar ${ }^{2}$ \\ ${ }^{1}$ Department of Mathematics, Ponnaiyah Ramajayam Institute of Science \& Technology (PRIST) University, \\ Vallam - 613 403, Thanjavur(Dt), Tamil Nadu, India \\ ${ }^{2}$ Department of Mathematics, A.V.V.M. Sri Pushpam College (Autonomous), \\ Thanjavur (Dt), Tamil Nadu, India \\ *Corresponding authorE-mail: hari.math3@gmail.com
}

Copyright $\odot 2014$ A. Hari Ganesh S. Jayakumar. This is an open access article distributed under the Creative Commons Attribution License, which permits unrestricted use, distribution, and reproduction in any medium, provided the original work is properly cited.

\begin{abstract}
Ranking of fuzzy numbers play an important role in decision making, optimization, forecasting etc. fuzzy numbers must be ranked before an action is taken by a decision maker. In this paper we propose a new approach of the fuzzy ranking of generalized trapezoidal fuzzy numbers based on radius of gyration point of centroids. The main advantage of the proposed approach is that the proposed approach provides the correct ordering of generalized and normal trapezoidal fuzzy numbers. We have also used some comparative examples to illustrate the advantage of the proposed approach for ranking of fuzzy numbers.
\end{abstract}

Keywords: Generalized trapezoidal fuzzy numbers, centroid, and radius of gyration.

\section{Introduction}

Ranking of fuzzy numbers is an important and prerequisite procedure for decision makers in many applications such as fuzzy risk analysis, fuzzy optimization etc. First method for ranking fuzzy numbers was introduced by Jain [7] in the year 1976, then a large number of methods have been developed to rank fuzzy numbers some of them have been reviewed and compared by Bortolan \& Degani [1], Chen \& Hwang [2] and Wang \& Kerre [15]. Among the existing ranking methods of fuzzy numbers, a number of them are focus on the centroid point and its distance from origin or the area measurement for the fuzzy numbers. Yagar[18] is a first researcher, who contributed the centroid concept in ranking method. Murakami [9], who is the first researcher, presented both horizontal X and Y coordinates of the centroid - point as the ranking index in 1983. Chu and Tsao have proposed a centroid index ranking method that calculates the distance between the centroid point of each fuzzy number and the original point to improve some ranking methods [5]. Cheng [3] proposed a distance index which is based on both horizontal and vertical coordinate of the centroid point. Wang et al [16] proposed the formula for finding horizontal and vertical coordinates of centroid point. Shief [12] proposed the correct formula for finding horizontal and vertical coordinates of centroid point. Wang \& Lee [17] proposed the ranking index on horizontal or vertical coordinates of centroid. Yong \& Liu [19] used radius of gyration with respect to horizontal axis and vertical axis as the coordinates of centroid point in area based ranking method. Recently, Phani Bushan Rao and others [10], [11], [14], [13] have proposed the formula for finding center of gravity point using circumcenter of centroids, orthocenter of centroids, incenter of centroids and centroid of centroids. In this paper a new method is proposed which is based on radius of gyration of centroids to rank fuzzy quantities. In a trapezoidal fuzzy number, first the trapezoid is split into three parts where the first, second and third parts are a triangle, a rectangle and a triangle respectively. Then the centroids of these parts are calculated followed by the calculation of the radius of gyration of these centroids. Most of the ranking procedures proposed in literature used centroid of trapezoid as reference point, as the centroid is a balancing point of trapezoid. But the radius of gyration of centroids can be considered a much more balancing point than the centroid. The main advantage of the proposed approach is that the proposed approach provides the correct ordering of generalized trapezoidal normal and non - normal fuzzy numbers 
and also the proposed approach is very simple and easy to apply in the real life problems. For the validation, the results of the proposed approach are compared with different existing approaches.

The rest of the paper is organized as follows: Section 2 introduces basic concepts and definitions of fuzzy numbers. Section 3 proposes an approach to ranking generalized trapezoidal fuzzy numbers based on radius of gyration and centroid. Section 4 presents four numerical examples to illustrate the advantages of the proposed approach. The paper ends with conclusion in Section 5.

\section{Preliminaries}

In this section, we introduce the basic concepts of fuzzy numbers, centroid point of fuzzy numbers and the radius of gyration (ROG) point of fuzzy numbers, which are play an important role in our proposed method.

\subsection{Fuzzy numbers}

Let $\mathrm{R}$ be the set of all real numbers. We assume a fuzzy number A that can be expressed for all $x \in R$ in the form

$$
A(x)=\left\{\begin{array}{lc}
A_{L}(x) & a \leq x \leq b \\
w & b \leq x \leq c \\
A_{R}(x) & c \leq x \leq d \\
0 & \text { otherwise }
\end{array}\right.
$$

Where $0 \leq w \leq 1$ is a constant, a, b, c, d are real numbers, such that a b b c $<\mathrm{d}$, $A_{L}(x):[a, b] \rightarrow[0, w], A_{R}(x):[c, d] \rightarrow[0, w]$ are two strictly monotonic and continuous functions from $R$ to the close interval $[0, \mathrm{w}]$.

Since $A_{L}(x)$ is continuous and strictly increasing, the inverse function of $A_{L}(x)$ exists. Similarly $A_{R}(x)$ is continuous and strictly decreasing, the inverse function of $A_{R}(x)$ also exist. The inverse functions of $A_{L}(x)$ and $A_{R}(x)$ can be denoted by $A_{L}^{-1}(x)$ and $A_{R}^{-1}(x)$, respectively. $A_{L}^{-1}(x)$ and $A_{R}^{-1}(x)$ are continuous on $[0, w]$ that means both $\int_{0}^{w} A_{L}^{-1}(x)$ and $\int_{0}^{\mathrm{w}} \mathrm{A}_{\mathrm{R}}^{-1}(\mathrm{x})$ exist.

\section{Definition}

A fuzzy number $\mathrm{A}=(\mathrm{a}, \mathrm{b}, \mathrm{c}, \mathrm{d})$ is called a trapezoidal fuzzy number if its membership function $\mathrm{A}(\mathrm{x})$ has the following form:

$A(x)= \begin{cases}\frac{w(x-a)}{b-a} & a \leq x \leq b \\ w & b \leq x \leq c \\ \frac{w(d-x)}{d-c} & c \leq x \leq d \\ 0 & \text { otherwise }\end{cases}$

In this case (see fig.1), the inverse functions $\mathrm{A}_{\mathrm{L}}^{-1}(\mathrm{x})$ and $\mathrm{A}_{\mathrm{R}}^{-1}(\mathrm{x})$ can be expressed analytically as

$$
\begin{array}{lc}
\mathrm{A}_{\mathrm{L}}^{-1}(\mathrm{x})=\mathrm{a}+(\mathrm{b}-\mathrm{a}) / \mathrm{w} & 0 \leq \mathrm{y} \leq \mathrm{w} \\
\mathrm{A}_{\mathrm{R}}^{-1}(\mathrm{x})=\mathrm{d}-(\mathrm{d}-\mathrm{c}) / \mathrm{w} & 0 \leq \mathrm{y} \leq \mathrm{w}
\end{array}
$$

\subsection{The centroid point of fuzzy numbers}

The centroid point of a fuzzy number A, Cheng [3] provided the following centroid formulae: 


$$
\begin{aligned}
\tilde{x}(A) & =\frac{\int_{a}^{b}\left(x A_{L}(x)\right) d x+\int_{b}^{c}(x) d x+\int_{c}^{d}\left(x A_{R}(x)\right) d x}{\int_{a}^{b}\left(A_{L}(x)\right) d x+\int_{b}^{c} d x+\int_{c}^{d}\left(A_{R}(x)\right) d x} \\
\tilde{y}(A) & =\frac{\int_{0}^{w}\left(y A_{L}^{-1}(y)\right) d y+\int_{0}^{w}\left(y A_{R}^{-1}(y)\right) d y}{\int_{0}^{w}\left(A_{L}^{-1}(y)\right) d y+\int_{0}^{w}\left(A_{R}^{-1}(y)\right) d y}
\end{aligned}
$$

Where $A_{L}$ and $A_{R}$ are the left and right membership functions of the fuzzy number $A \cdot A_{L}^{-1}(x)$ and $A_{R}^{-1}(x)$ are the inverse functions of $A_{L}$ and $A_{R}$, respectively.

Normal fuzzy numbers can be obtained as special cases of non-normal fuzzy numbers with w=1.

\subsection{The radius of gyration of fuzzy numbers}

Radius of gyration (ROG) is a concept in mechanics. The ROG point $\left(r_{x}, r_{y}\right)$ for a fuzzy number $\tilde{A}$ is provided as [19]

$r_{x}=\sqrt{\frac{I_{x}(\tilde{A})}{A(\tilde{A})}}$

$r_{y}=\sqrt{\frac{I_{y}(\tilde{A})}{A(\tilde{A})}}$

Where $I_{x}(\tilde{A})$ and $I_{y}(\tilde{A})$ are moment of inertia of $\tilde{A}$ with respect to $x$ and $y$ axis respectively and $A(\tilde{A})$ is the area of $\tilde{A}$.

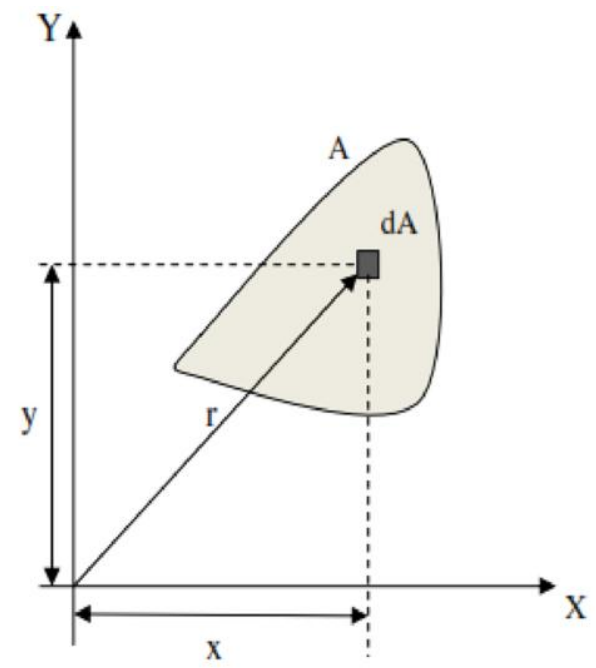

Fig. 1: The moment of inertia of an area

\section{The Proposed approach to ranking fuzzy numbers}

The centroid of a trapezoidal is considered as the balancing point of the trapezoid (Figure 2). Divide the trapezoid into three plane figures. The plane figures are a triangle (APB), a rectangle (BPQC), and triangle (CQD), respectively. The radius of gyration of the centroids of these plane figures is taken as the point of reference to define the ranking of generalized fuzzy members. The reason for selecting this point as a point of reference is that each centroid point (G1 of triangle $\mathrm{APB}, \mathrm{G} 2$ of rectangle $\mathrm{BPQC}$, and G3 of triangle CQD) are balancing points of each individual plane figure, and the ROG of these centroid points is much more balancing point for a generalized trapezoidal fuzzy numbers. Therefore, this point would be a better reference point than the centroid point of a trapezoid.

Consider a generalized trapezoidal fuzzy number $\mathrm{A}=(\mathrm{a}, \mathrm{b}, \mathrm{c}, \mathrm{d}$; w) (Figure 2). The centroids of the three plane figures are $\mathrm{G}_{1}=\left(\frac{(\mathrm{a}+2 \mathrm{~b})}{3}, \frac{\mathrm{w}}{3}\right), \mathrm{G}_{2}=\left(\frac{(\mathrm{b}+\mathrm{c})}{2}, \frac{\mathrm{w}}{2}\right), \mathrm{G}_{3}=\left(\frac{(2 \mathrm{c}+\mathrm{d})}{3}, \frac{\mathrm{w}}{3}\right)$ respectively. Equation of the line $\overline{G_{1} G_{3}}$ is $\mathrm{y}=\mathrm{w} / 3$ and G2 does not lie on the line $\overline{G_{1} G_{3}}$. Therefore, G1, G2 and G3 are non - collinear and they form a triangle.

We define the radius of gyration point of a triangle with vertices G1, G2 and G3 of the generalized trapezoidal fuzzy number 
$\tilde{A}=(a, b, c, d ; w)$

As

$\left(r_{x}^{\tilde{A}}, r_{y}^{\tilde{A}}\right)=\left(\sqrt{\frac{(2(c-b)+(d-a))^{2}}{162}+\frac{(2(a+d)+7(b+c))^{2}}{324}}, \sqrt{\frac{11}{72}} w\right)$

As a special case, for triangular fuzzy number

$\tilde{A}=(a, b, d ; w)$

That is, $c=b$ the radius of gyration point of centroids is given by

$\left(r_{x}^{\tilde{A}}, r_{y}^{\tilde{A}}\right)=\left(\sqrt{\frac{(d-a)^{2}}{162}+\frac{(2(a+d)+14 b)^{2}}{324}}, \sqrt{\frac{11}{72}} w\right)$

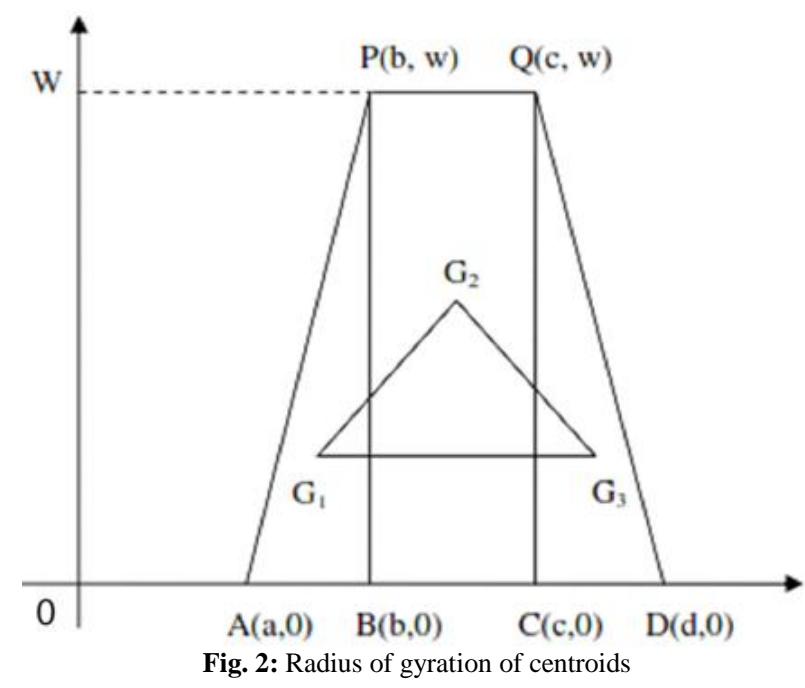

Now $A=\left(a_{1}, b_{1}, c_{1}, d_{1} ; w_{1}\right)$ and $B=\left(a_{2}, b_{2}, c_{2}, d_{2} ; w_{2}\right)$ to two generalized trapezoidal fuzzy numbers. The working procedure to compare $\mathrm{A}$ and $\mathrm{B}$ is as follows:

Find $r_{y}(A)$ and $r_{y}(B)$

Case (i): If $r_{x}(A)>r_{x}(B) \Rightarrow A>B$

Case (ii): If $r_{x}(A)<r_{x}(B) \Rightarrow A<B$

Case (iii): If $r_{x}(A)=r_{x}(B) \Rightarrow$

$\left\{\begin{array}{l}r_{y}(A)>r_{y}(B) \Rightarrow A>B \\ r_{y}(A)<r_{y}(B) \Rightarrow A<B \\ r_{y}(A)=r_{y}(B) \Rightarrow A=B\end{array}\right.$

\section{Result and discussion}

In this section, the proposed method is compared with other existing methods to show the validity and advantage.

Example 1:

The two triangular fuzzy numbers $\mathrm{A}=(1,4,5), \mathrm{B}=(2,3,6)$ are ranked by our method.

Proposed method concludes that:

$\left(\mathrm{r}_{\mathrm{x}}(\mathrm{A}), \mathrm{r}_{\mathrm{y}}(\mathrm{A})\right)=(3.790,0.3909)$

$\left(\mathrm{r}_{\mathrm{x}}(\mathrm{B}), \mathrm{r}_{\mathrm{y}}(\mathrm{B})\right)=(2.237,0.3909)$

Therefore,

$\mathrm{r}_{\mathrm{x}}(\mathrm{A})>\mathrm{r}_{\mathrm{x}}(\mathrm{B}) \Rightarrow \mathrm{A}>\mathrm{B}$

By Liou and Wang Method [8], it is clear that the two fuzzy numbers are equal for all the decision makers as

$I_{T}^{\alpha}(A)=4.5 \alpha+(1-\alpha) 2.5$

$I_{T}^{\alpha}(B)=4.5 \alpha+(1-\alpha) 2.5$ 


\section{Example 2:}

Let $\mathrm{A}=(0.2,0.2,0.2,0.2 ; 1)$ and $\mathrm{B}=(1,1,1,1 ; 1)$ be two crisp numbers which are the special case of fuzzy numbers. Chang [3] ranked fuzzy numbers with distance method using the Euclidean distance between centroid point and orginal point. Whereas Chu \& Tsao [5] proposed a ranking function which is the area between the centroid point and original point. Their centroid formula are given by

$$
\begin{aligned}
& \left(\bar{x}_{0}, \bar{y}_{0}\right)=\left(\frac{w\left(d^{2}-2 c^{2}+2 b^{2}-a^{2}+d c-a b\right)+3\left(c^{2}-b^{2}\right)}{3 w(d-c+b-a)+6(c-b)}, \frac{\mathrm{w}}{3}\left(1+\frac{(b+c)-(a+d)(1-w)}{(b+c-a-d)+2(a+d) w}\right)\right) \\
& \left(\bar{x}_{0}, \bar{y}_{0}\right)=\left(\frac{w\left(d^{2}-2 c^{2}+2 b^{2}-a^{2}+d c-a b\right)+3\left(c^{2}-b^{2}\right)}{3 w(d-c+b-a)+6(c-b)}, \frac{\mathrm{w}}{3}\left(1+\frac{(b+c)}{a+b+c+d}\right)\right)
\end{aligned}
$$

Both these centroid formulae cannot rank crisp numbers as it can be seen from the above formulae that the denominator in the first coordinate of their centroid formula is zero, and hence centroid of crisp numbers are undefined for their formulae.

Proposed method shows that

$\left(\mathrm{r}_{\mathrm{x}}(\mathrm{A}), \mathrm{r}_{\mathrm{y}}(\mathrm{A})\right)=(0.2,0.3909)$

$\left(r_{\mathrm{x}}(\mathrm{B}), \mathrm{r}_{\mathrm{y}}(\mathrm{B})\right)=(1,0.3909)$

Since $r_{x}(A)<r_{x}(B) \Rightarrow A<B$

\section{Example 3:}

Let $\mathrm{A}=(3,4,5,6: 1)$ and $\mathrm{B}=(7,8,9,10 ; 0.2)$ be two fuzzy numbers.

Yong \& Liu [19] ranked fuzzy numbers with area method using the area between ROG point and original point. The ROG point of generalized trapezoidal fuzzy number $(\mathrm{a}, \mathrm{b}, \mathrm{c}, \mathrm{d} ; \mathrm{w})$ is given by

$$
\left(r_{x}, r_{y}\right)=\left(\sqrt{\frac{\left(I_{x}\right)_{1}+\left(I_{x}\right)_{2}+\left(I_{x}\right)_{3}}{(((c-b)+(d-a)) \cdot w / 2}}, \sqrt{\frac{\left(I_{y}\right)_{1}+\left(I_{y}\right)_{2}+\left(I_{y}\right)_{3}}{(((c-b)+(d-a)) \cdot w / 2}}\right)
$$

Where they $\left(I_{x}\right)_{1},\left(I_{x}\right)_{2},\left(I_{x}\right)_{3},\left(I_{y}\right)_{1},\left(I_{y}\right)_{2},\left(I_{y}\right)_{3}$ are moment of intertia of the three shapes 1,2 and 3 (fig).

By ROG method, we can calculate,

$\left(\mathrm{r}_{\mathrm{x}}(\mathrm{A}), \mathrm{r}_{\mathrm{y}}(\mathrm{A})\right)=(0.5,6.7082)$

$\left(r_{\mathrm{x}}(\mathrm{B}), \mathrm{r}_{\mathrm{y}}(\mathrm{B})\right)=(0.1,15.2407)$

Therefore,

$\left(\mathrm{r}_{\mathrm{x} \times \mathrm{r}} \mathrm{r}_{\mathrm{y}}\right)(\mathrm{A})=3.3541$

$\left(\mathrm{r}_{\mathrm{x} \times \mathrm{r}} \mathrm{r}_{\mathrm{y}}\right)(\mathrm{B})=1.5241$

According to the ROG method, it is shown that A is bigger than B. However, A should be smaller than B intuitively Proposed method shows that

$\left(\mathrm{r}_{\mathrm{x}}(\mathrm{A}), \mathrm{r}_{\mathrm{y}}(\mathrm{A})\right)=(4.5171,0.3909)$

$\left(r_{x}(B), r_{y}(B)\right)=(8.5091,0.0782)$

Since $r_{x}(A)<r_{x}(B) \Rightarrow A<B$

\section{Example 4:}

Consider four fuzzy numbers follows:

$\mathrm{A} 1=(0.1,0.2,0.3 ; 1), \mathrm{A} 2=(0.2,0.5,0.8 ; 1), \mathrm{A} 3=(0.3,0.4,0.9 ; 1), \mathrm{A} 4=(0.6,0.7,0.8 ; 1)$

\begin{tabular}{|c|c|c|c|c|c|c|}
\hline \multicolumn{2}{|c|}{ Fuzzy Numbers } & A1 & $\mathrm{A} 2$ & $\mathrm{~A} 3$ & $\mathrm{~A} 4$ & Ranking Order \\
\hline \multicolumn{2}{|c|}{ Yager [18 ] } & 0.2 & 0.5 & 0.5 & 0.7 & $\mathrm{~A}_{4}>\mathrm{A}_{2} \approx \mathrm{A}_{3}>\mathrm{A}_{1}$ \\
\hline \multicolumn{2}{|c|}{ Fortemps \& Roubens [6] } & 0.2 & 0.5 & 0.5 & 0.7 & $\mathrm{~A}_{4}>\mathrm{A}_{2} \approx \mathrm{A}_{3}>\mathrm{A}_{1}$ \\
\hline \multirow{3}{*}{$\begin{array}{c}\text { Liou \& } \\
\text { Wang } \\
{[8]}\end{array}$} & $\alpha=1$ & 0.25 & 0.65 & 0.65 & 0.75 & $\mathrm{~A}_{4}>\mathrm{A}_{2} \approx \mathrm{A}_{3}>\mathrm{A}_{1}$ \\
\hline & $\alpha=0.5$ & 0.2 & 0.5 & 0.5 & 0.7 & $\mathrm{~A}_{4}>\mathrm{A}_{2} \approx \mathrm{A}_{3}>\mathrm{A}_{1}$ \\
\hline & $\alpha=0$ & 0.15 & 0.35 & 0.35 & 0.65 & $\mathrm{~A}_{4}>\mathrm{A}_{2} \approx \mathrm{A}_{3}>\mathrm{A}_{1}$ \\
\hline \multirow{3}{*}{ Chen [4] } & $\beta=1$ & -0.2 & 0.0 & 0.0 & -0.2 & $\mathrm{~A}_{2} \approx \mathrm{A}_{3}>\mathrm{A}_{1} \approx \mathrm{A}_{4}$ \\
\hline & $\beta=0.5$ & -0.2 & 0.0 & 0.0 & -0.2 & $\mathrm{~A}_{2} \approx \mathrm{A}_{3}>\mathrm{A}_{1} \approx \mathrm{A}_{4}$ \\
\hline & $\beta=0$ & -0.2 & 0.0 & 0.0 & -0.2 & $\mathrm{~A}_{2} \approx \mathrm{A}_{3}>\mathrm{A}_{1} \approx \mathrm{A}_{4}$ \\
\hline \multicolumn{2}{|c|}{ Proposed Method } & 0.2006 & 0.5022 & 0.4469 & 0.7002 & $A_{4}>A_{2}>A_{3}>A_{1}$ \\
\hline
\end{tabular}

The result of ranking of above four fuzzy numbers given in Table 1

Table 1: Comparative result of Example 4 


\section{Conclusion}

In this paper, a new procedure of ranking fuzzy numbers using radius of gyration and centroid is proposed. This method ranks trapezoid as soon as triangular fuzzy numbers. This method also ranks crisp numbers which are special case of fuzzy numbers. Comparative examples are used to illustrate the advantages of the proposed method. Additionally, the proposed approach can provide decision makers with a new alternative to rank fuzzy numbers.

\section{References}

[1] Bortolan, G., \& Degani, R. A review of some methods for ranking fuzzy subsets, Fuzzy Sets and Systems, 15 (1), 1 - 99 , 1985

[2] Chen, S.J., \& Hwang, C.L. Fuzzy Multiple Attribute Decision Making, Springer, Berlin, 1992.

[3] Cheng, C. H. A New approach for ranking fuzzy numbers by distance method. Fuzzy Sets and Systems, 95, $307-317,1998$.

[4] Chen, S. Ranking fuzzy numbers with maximizing set and minimizing set, Fuzzy Sets and Systems, 17, 113 - 129, (1985).

[5] Chu, T. \& Tsao, C. Ranking fuzzy numbers with an area between the centroid point and original point, Computers and Mathematics with Applications, 43, $111-117,2002$.

[6] Fortemps, P. and Roubens, M. Ranking and defuzzification methods based on area compensation, Fuzzy Sets and Systems, 82, 319 - 330, 1996.

[7] Jain, R. Decision making in the presence of fuzzy variable. IEEE Transactions on Systems Man, and Cybrenctics, 6, 698 - 703, 1976

[8] Liou, T.S. and Wang, M.J., Ranking fuzzy numbers with integral value, Fuzzy Sets and Systems, 50, 247 - 255, (1992).

[9] Murakami, S. et al., Fuzzy decision analysis on the development of centralized regional energy control system, in: IFAC symposium on Fuzzy Information Knowledge Representation and Decision Analysis pp.363 - 368, 1983

[10] Phani Bushan Rao \& Ravi Shankar, N. Ranking Fuzzy Numbers with a Distance Method using Circumcenter of centroids and an Index Modality, Advances in Fuzzy Systems, pp. 1 -7, 2011.

[11] Ravi Shankar. N., et al., Fuzzy Risk Analysis based on A New Approach of Ranking Fuzzy Numbers using Orthocenter of Centroids, International Journal of Computer Applications, Vol. 42, No. 3, 24 - 36, 2012.

[12] Shieh. B.S. An approach to centroids of fuzzy numbers, International Journal of Fuzzy Systems, 9(1), 51 - 54, 2007.

[13] Suresh Babu. S., et al., Ranking Generalized Fuzzy Numbers using centroid of centroids, International Journal of Fuzzy Logic Systems (IJFLS), Vol2, No.3, $17-32,2012$.

[14] Thorani, Y.L.P. et al. Ordering Generalized Trapezoidal Fuzzy Numbers, International Journal of Contemporary Mathematical Sciences, Vol. 7 , no. 12 , pp. $555-573,2012$.

[15] Wang, X., \& Kerre, E.E. Reasonable properties for the ordering of fuzzy quantities (I), Fuzzy Sets and Systems, $118,375-385,2001$.

[16] Wang, Y.M. et al. On the centroids of fuzzy numbers, Fuzzy Sets and Systems, 157, 919 - 926, 2006.

[17] Wang, Y.J., \& Lee, H.S. The revised method of ranking fuzzy numbers with an area between the centroid and original points, Computers and Mathematics with applications, 55, 2033-2042, 2008

[18] Yager, R.R. A procedure for ordering fuzzy subsets of the unit interval, Information Sciences 24, 143 - 161, 1981

[19] Yong \& Liu. Ranking fuzzy numbers with an area method using radius of gyration, Computers and Mathematics with applications. 51, 1127 1136,2006 